\title{
Higgs Boson Pair Production at Next-to-Next-to-Leading Order in QCD
}

\author{
Daniel de Florian* and Javier Mazzitelli ${ }^{\dagger}$ \\ Departamento de Física, FCEyN, Universidad de Buenos Aires, (1428) Pabellón 1, Ciudad Universitaria, Capital Federal, Argentina
} (Received 25 September 2013; published 12 November 2013)

\begin{abstract}
We compute the next-to-next-to-leading order QCD corrections for standard model Higgs boson pair production inclusive cross section at hadron colliders within the large top-mass approximation. We provide numerical results for the LHC, finding that the corrections are large, resulting in an increase of $\mathcal{O}(20 \%)$ with respect to the next-to-leading order result at c.m. energy $\sqrt{s_{H}}=14 \mathrm{TeV}$. We observe a substantial reduction in the scale dependence, with overlap between the current and previous order prediction. All our results are normalized using the full top- and bottom-mass dependence at leading order. We also provide analytical expressions for the $K$ factors as a function of $s_{H}$.
\end{abstract}

DOI: 10.1103/PhysRevLett.111.201801

PACS numbers: $14.80 . \mathrm{Bn}, 12.38 . \mathrm{Bx}$

Introduction.-The recent discovery of a new boson $[1,2]$, so far compatible with the long sought standard model (SM) Higgs boson [3], at the Large Hadron Collider (LHC) opens a new stage in the task of understanding the mechanism of electroweak symmetry breaking. In order to determine the connection between this phenomenon and the new particle, it is crucial to measure its couplings to gauge bosons, fermions, and its self-interactions. In particular, the knowledge of the Higgs self-couplings is the only way to reconstruct the scalar potential.

Higgs trilinear coupling can be studied via Higgs pair production. Recently, several papers have analyzed the possibility of measuring this process at the LHC [4-12]. In general, it has been shown that despite the smallness of the signal and the large background, its measurement can be achieved at a luminosity upgraded LHC. For example, for $b \bar{b} \gamma \gamma$ and $b \bar{b} \tau^{+} \tau^{-}$final states, after the application of proper cuts, the significances obtained are $\sim 16$ and $\sim 9$, respectively, for a c.m. energy of $14 \mathrm{TeV}$ and an integrated luminosity of $3000 \mathrm{fb}^{-1}$ [7]. These are, so far, the most promising final states for the Higgs trilinear coupling analysis. The sensitivity of these channels can be further improved by the application of jet substructure techniques, as was shown in Refs. [5,6,12].

The SM Higgs pair production at hadron colliders is dominated by the gluon fusion mechanism mediated by a heavy-quark loop. At leading order (LO) in QCD perturbation theory, this process can occur either through a box $g g \rightarrow H H$ or a triangle $g g \rightarrow H^{*} \rightarrow H H$ diagram, of which only the latter is sensitive to the Higgs trilinear coupling. This cross section has been calculated in Refs. [13-15]. The QCD next-to-leading order (NLO) corrections, within the large top-mass $\left(M_{t}\right)$ approximation, have been computed in Ref. [16], finding an inclusive $K$ factor close to 2 . The size of this correction makes it essential to reach higher orders to be able to provide accurate theoretical predictions.

Recently, the two-loop corrections were calculated by us in Ref. [17], again in the large top-mass limit, and the next-to-next-to-leading order (NNLO) cross section was evaluated within the soft-virtual approximation, following the results of Ref. [18]. We found an increase close to $23 \%$ with respect to the NLO result.

The finite top-mass effects were analyzed at NLO in Ref. [19], finding that the accuracy of the large top-mass approximation at NLO is dramatically improved if the exact top-mass leading order cross section is used to normalize the corrections, achieving a precision of $\mathcal{O}(10 \%)$.

In this Letter, we present the full NNLO corrections for the inclusive cross section in the large top-mass limit. We also provide numerical predictions for the LHC, using the exact leading order result to normalize the partonic cross section.

Results.-Within the large top-mass approximation, the effective single and double-Higgs coupling to gluons is given by the following Lagrangian

$$
\mathcal{L}_{\text {eff }}=-\frac{1}{4} G_{\mu \nu} G^{\mu \nu}\left(C_{H} \frac{H}{v}-C_{H H} \frac{H^{2}}{v^{2}}\right),
$$

where $G_{\mu \nu}$ stands for the gluonic field strength tensor and $v \simeq 246 \mathrm{GeV}$ is the Higgs vacuum expectation value. While the $\mathcal{O}\left(\alpha_{\mathrm{S}}^{3}\right)$ of the $C_{H}$ expansion is known [20,21], the QCD corrections of $C_{H H}$ are only known up to $\mathcal{O}\left(\alpha_{\mathrm{S}}^{2}\right)$ [22]. Up to that order, both expansions yield the same result. Even when this approximation is not reliable at $\mathrm{LO}$, it is a very accurate mechanism for the computation of the higher order corrections if the exact LO result is used, since QCD corrections are dominated by soft contributions which are not affected by the details of the effective vertex.

To compute the SM Higgs boson pair production cross section to NNLO accuracy, we need to evaluate the QCD perturbative expansion up to $\mathcal{O}\left(\alpha_{\mathrm{S}}^{4}\right)$. We will separate the contributions to the squared matrix element into two classes: (a) those containing two gluon-gluon-Higgs vertices (either $g g H$ or $g g H H$ ) and (b) those containing three or four effective vertices. Then the partonic cross section will be written as 


$$
Q^{2} \frac{d \hat{\sigma}}{d Q^{2}}=\hat{\sigma}^{a}+\hat{\sigma}^{b}
$$

where $Q^{2}$ is the squared invariant mass of the Higgs pair system. For the sake of completeness we also include the LO and NLO contributions in $\hat{\sigma}^{a}$ and $\hat{\sigma}^{b}$.

Contributions to $\hat{\sigma}^{a}$ only contain diagrams with one effective vertex each. Given the similarity between $g g H$ and $g g H H$ vertices, the corrections are equal to those of single Higgs production [23-25] up to an overall LO normalization. Specifically, for each partonic subprocess $i j \rightarrow H H+X$ we have (for factorization and renormalization scales $\mu_{F}=\mu_{R}=Q$ )

$$
\begin{aligned}
\hat{\sigma}_{i j}^{a}= & \hat{\sigma}_{\mathrm{LO}}\left\{\eta_{i j}^{(0)}+\left(\frac{\alpha_{\mathrm{S}}}{2 \pi}\right) 2 \eta_{i j}^{(1)}+\left(\frac{\alpha_{\mathrm{S}}}{2 \pi}\right)^{2}\left[4 \eta_{i j}^{(2)}\right.\right. \\
& \left.\left.+8 \delta_{i g} \delta_{j g} \delta(1-x) \frac{\operatorname{Re}\left(C_{\mathrm{LO}}\right)}{\left|C_{\mathrm{LO}}\right|^{2}}\left(C_{H}^{(2)}-C_{H H}^{(2)}\right)\right]\right\},
\end{aligned}
$$

where

$$
\hat{\sigma}_{\mathrm{LO}}=\int_{t_{-}}^{t_{+}} d t \frac{G_{F}^{2} \alpha_{\mathrm{S}}^{2}}{512(2 \pi)^{3}}\left\{\left|C_{\triangle} F_{\triangle}+C_{\square} F_{\square}\right|^{2}+\left|C_{\square} G_{\square}\right|^{2}\right\},
$$

and, for the sake of brevity, we refer the reader to Ref. [24] for the expressions of $\eta_{i j}$ and to Ref. [15] for $C_{\triangle}, F_{\triangle}, C_{\square}$, $F_{\square}$, and $G_{\square}$. The term proportional to $\delta_{i g} \delta_{j g}$ in Eq. (3) arises from a possible difference between the second order corrections to the vertices $g g H$ and $g g H H$, of which the latter is still unknown $\left(C_{H H}^{(2)}\right.$ and $C_{H}^{(2)}$ are defined as in Ref. [17]). The exact LO partonic cross section $\hat{\sigma}_{\text {LO }}$ depends on $Q^{2}$ and $t$, the latter given by

$$
t=-\frac{1}{2}\left(Q^{2}-2 M_{H}^{2}-Q \sqrt{Q^{2}-4 M_{H}^{2}} \cos \theta_{1}\right),
$$

where $\theta_{1}$ is the scattering angle in the Higgs pair c.m. system and the integration limits $t_{ \pm}$correspond to $\cos \theta_{1}=$ \pm 1 . In the large top mass limit $\hat{\sigma}_{\mathrm{LO}}$ takes the following simple form,

$$
\hat{\sigma}_{\mathrm{LO}}=\int_{t_{-}}^{t_{+}} d t\left(\frac{\alpha_{\mathrm{S}}}{2 \pi}\right)^{2} F_{\mathrm{LO}}\left|C_{\mathrm{LO}}\right|^{2},
$$

where

$$
F_{\mathrm{LO}}=\frac{G_{F}^{2}}{2304 \pi}, \quad C_{\mathrm{LO}}=\frac{3 M_{H}^{2}}{Q^{2}-M_{H}^{2}+i M_{H} \Gamma_{H}}-1 .
$$

Here $\Gamma_{H}$ stands for the Higgs total width, while $G_{F}$ is the Fermi coupling.

Since each $g g H$ and $g g H H$ vertex is proportional to $\alpha_{\mathrm{S}}$, contributions to $\hat{\sigma}^{b}$ first appear at NLO, as a tree-level contribution to the subprocess $g g \rightarrow H H$. Then at NNLO we have one-loop and single real emission corrections. The former have been calculated in Ref. [17]. Specifically, they are all the terms of Eq. (8) of Ref. [17] which are not proportional to $\left|C_{\mathrm{LO}}\right|^{2}$, except for the term proportional to
$\left(C_{H}^{(2)}-C_{H H}^{(2)}\right)$ which we have already moved into $\hat{\sigma}^{a}$. We will denote this contribution by $\hat{\sigma}^{(v)}$.

Finally, the only remaining part of the NNLO contribution to the cross section arises from the real emission processes present in $\hat{\sigma}^{b}$, which we will denote by $\hat{\sigma}^{(r)}$. The partonic subprocesses involved are $g g \rightarrow H H+g$ and $q g \rightarrow H H+q$ (with the corresponding crossings). Examples of the Feynman diagrams involved in the calculation are shown in Fig. 1.

To compute this contribution we used the MATHEMATICA packages FEYNARTS [26] and FEYNCALC [27] in order to generate the Feynman diagrams and evaluate the corresponding amplitudes. The calculation was performed using nonphysical polarizations, which we cancelled including ghosts in the initial and final states. The results for $n=4$ dimensions can be found in the Supplemental Material [28]. In order to subtract the soft and collinear divergencies, we used the Frixione, Kunszt, and Signer subtraction method [29]. Below, we provide the details of the calculation.

Let $p_{1}$ and $p_{2}$ be the momenta of the incoming partons, $k_{1}$ and $k_{2}$ the momenta of the Higgs bosons and $k$ the momentum of the outgoing parton. We define the variables $x$ and $y$, where $x=Q^{2} / s$ and $y$ is the cosine of the angle between $p_{1}$ and $k$. In terms of these variables, soft singularities correspond to the limit $x \rightarrow 1$, while collinear singularities arise when $y \rightarrow \pm 1$. Gluon initiated subprocesses contain the three kinds of singularities while those processes initiated by quark-gluon are only affected by a collinear singularity and quark-antiquark ones are finite.

The three-particle phase space (PS) in $n=4-2 \epsilon$ dimensions is given by

$$
\begin{aligned}
d \mathrm{PS}_{3}= & (4 \pi)^{-2+\epsilon} \frac{\Gamma(1-\epsilon)}{\Gamma(1-2 \epsilon)} d \mathrm{PS}_{2}^{(x)} \frac{s^{1-\epsilon}}{2 \pi} \\
& \times(1-x)^{1-2 \epsilon}\left(1-y^{2}\right)^{-\epsilon} d y \sin ^{-2 \epsilon} \theta_{2} d \theta_{2},
\end{aligned}
$$

where $d \mathrm{PS}_{2}^{(x)}$ is obtained from the two-particle phase space through the replacement $s \rightarrow x s$, that is
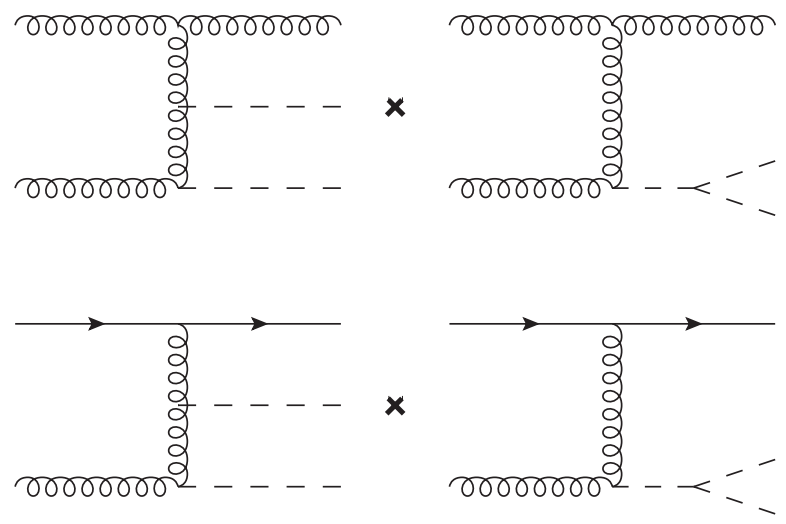

FIG. 1. Example of Feynman diagrams needed for the NNLO calculation for $\mathrm{gg} \rightarrow \mathrm{HHg}$ (top) and $q \mathrm{~g} \rightarrow \mathrm{HHq}$ (bottom) subprocesses. Other parton subprocesses can be obtained from crossings. 


$$
\begin{aligned}
d \mathrm{PS}_{2}^{(x)}= & \frac{(16 \pi)^{-1+\epsilon}}{\Gamma(1-\epsilon)}(x s)^{-\epsilon}\left(1-\frac{4 M_{H}^{2}}{x s}\right)^{(1 / 2)-\epsilon} \\
& \times \sin ^{-2 \epsilon} \theta_{1} d \cos \theta_{1} d x .
\end{aligned}
$$

The variables $\theta_{1}$ and $\theta_{2}$ are the polar and azimuthal angles of the Higgs boson with momentum $k_{1}$ in the center of mass frame of the Higgs pair system, and both of them range between 0 and $\pi$. All the relevant invariants for the process can be expressed in terms of $x, y, \theta_{1}$, and $\theta_{2}$ (see the Supplemental Material [28]). For more details about this parametrization, see, for example, Ref. [30].

We will focus now on the $g g \rightarrow H H+g$ subprocess since it suffers from all kinds of singularities. In the soft limit, the squared matrix element has a divergent behavior proportional to $(1-x)^{-2}$, while in the collinear limits it goes like $\left(1-y^{2}\right)^{-1}$. Combining those factors with the ones coming from the phase space, there is an overall factor $(1-x)^{-1-2 \epsilon}\left(1-y^{2}\right)^{-1-\epsilon}$ which regularizes all the divergences. The key to isolating the singularities, then, is to perform the $\epsilon$ expansion of that factor in the following way [30]:

$$
\begin{aligned}
&(1-x)^{-1-2 \epsilon}\left(1-y^{2}\right)^{-1-\epsilon} \\
&=-\frac{1}{2 \epsilon} \delta(1-x)\left(1-y^{2}\right)^{-1-\epsilon}-\frac{2^{-2 \epsilon}}{2 \epsilon}[\delta(1-y) \\
&+\delta(1+y)]\left[\left(\frac{1}{1-x}\right)_{+}-2 \epsilon\left(\frac{\log (1-x)}{1-x}\right)_{+}\right] \\
&+\frac{1}{2}\left(\frac{1}{1-x}\right)_{+}\left[\left(\frac{1}{1-y}\right)_{+}+\left(\frac{1}{1+y}\right)_{+}\right],
\end{aligned}
$$

where the plus distributions are defined as

$$
\begin{aligned}
& \int_{0}^{1} d x G_{+}(x) f(x)=\int_{0}^{1} d x G(x)[f(x)-f(1)] \\
& \int_{-1}^{1} d y f(y)\left(\frac{1}{1 \pm y}\right)_{+}=\int_{-1}^{1} d y \frac{f(y)-f(\mp 1)}{1 \pm y} .
\end{aligned}
$$

The delta functions in the first two terms of the expansion allow us to simplify considerably the complexity of the squared matrix element, leading to a much simpler analytical phase space integration. On the other hand, the last term in Eq. (10) is finite, and then, the integration can be performed (numerically) in four dimensions.

We directly present the final results. The gluon-gluon contribution to $\hat{\sigma}^{b}$ can be split in the following way:

$$
\hat{\sigma}_{g g}^{b}=\hat{\sigma}_{g g}^{(r)}+\hat{\sigma}^{(v)}=\hat{\sigma}_{g g}^{(s v)}+\hat{\sigma}_{g g}^{(c+)}+\hat{\sigma}_{g g}^{(c-)}+\hat{\sigma}_{g g}^{(f)},
$$

where the renormalized results (for $\mu_{F}=\mu_{R}=Q$ ) take the following form

$$
\begin{aligned}
\hat{\sigma}_{g g}^{(s v)}= & \frac{\hat{\sigma}_{\mathrm{LO}}}{\left|C_{\mathrm{LO}}\right|^{2}} \delta(1-x)\left\{\left(\frac{\alpha_{\mathrm{S}}}{2 \pi}\right) \frac{4}{3} \operatorname{Re}\left(C_{L O}\right)+\left(\frac{\alpha_{\mathrm{S}}}{2 \pi}\right)^{2}\right. \\
& \times\left[\operatorname{Re}\left(C_{\mathrm{LO}}\right)\left(\frac{8 \pi^{2}}{3}+\mathcal{R}^{(2)}-8\left(C_{H}^{(2)}-C_{H H}^{(2)}\right)\right)\right. \\
& \left.\left.+\operatorname{Im}\left(C_{\mathrm{LO}}\right) I^{(2)}+\mathcal{V}^{(2)}\right]\right\}, \\
\hat{\sigma}_{g g}^{(c+)}= & \hat{\sigma}_{g g}^{(c-)}=\frac{\hat{\sigma}_{\mathrm{LO}}}{\left|C_{\mathrm{LO}}\right|^{2}}\left(\frac{\alpha_{\mathrm{S}}}{2 \pi}\right)^{2} 8[1-(1-x) x]^{2} \\
& \times\left[2\left(\frac{\log (1-x)}{1-x}\right)_{+}-\frac{\log x}{1-x}\right] \operatorname{Re}\left(C_{\mathrm{LO}}\right), \\
\hat{\sigma}_{g g}^{(f)}= & \int d \cos \theta_{1} d \theta_{2} d y \frac{\sqrt{x\left(x-4 M_{H}^{2} / s\right)}}{1024 \pi^{4}}\left(\frac{1}{1-x}\right)_{+} \\
& \times\left[\left(\frac{1}{1-y}\right)_{+}+\left(\frac{1}{1+y}\right)_{+}\right] f_{g g}\left(x, y, \theta_{1}, \theta_{2}\right) .
\end{aligned}
$$

We have already included, in this expression, the counterterms arising from collinear factorization. The expressions for $\mathcal{R}^{(2)}, I^{(2)}$, and $\mathcal{V}^{(2)}$ can be found in Ref. [17]. We subtracted the term in $\mathcal{R}^{(2)}$ proportional to $C_{H}^{(2)}-C_{H H}^{(2)}$ since it has already been included in $\hat{\sigma}_{g g}^{a}$. The expression for $f_{g g}\left(x, y, \theta_{1}, \theta_{2}\right)$ can be found in the Supplemental Material [28]. We also included the NLO contribution to $\hat{\sigma}^{b}$ in the definition of $\hat{\sigma}_{g g}^{(s v)}$.

Using a similar procedure we obtain the results for the $q g$ and $g q$ channels ( $q$ stands for any massless quark or antiquark), which can be split into two contributions,

$$
\begin{aligned}
& \hat{\sigma}_{q g}^{b}=\hat{\sigma}_{q g}^{(r)}=\hat{\sigma}_{q g}^{(c+)}+\hat{\sigma}_{q g}^{(f),} \\
& \hat{\sigma}_{g q}^{b}=\hat{\sigma}_{g q}^{(r)}=\hat{\sigma}_{g q}^{(c-)}+\hat{\sigma}_{g q}^{(f)},
\end{aligned}
$$

which take the following form

$$
\begin{aligned}
\hat{\sigma}_{q g}^{(c+)}= & \hat{\sigma}_{g q}^{(c-)}=\frac{\hat{\sigma}_{\mathrm{LO}}}{\left|C_{\mathrm{LO}}\right|^{2}}\left(\frac{\alpha_{\mathrm{S}}}{2 \pi}\right)^{2} \frac{16}{9}\left\{\left[1+(1-x)^{2}\right]\right. \\
& \left.\times[2 \log (1-x)-\log x]+x^{2}\right\} \operatorname{Re}\left(C_{\mathrm{LO}}\right), \\
\hat{\sigma}_{q g}^{(f)}= & \int d \cos \theta_{1} d \theta_{2} d y \frac{\sqrt{x\left(x-4 M_{H}^{2} / s\right)}}{512 \pi^{4}} \\
& \times\left(\frac{1}{1-y}\right)_{+} f_{q g}\left(x, y, \theta_{1}, \theta_{2}\right), \\
\hat{\sigma}_{g q}^{(f)}= & \int d \cos \theta_{1} d \theta_{2} d y \frac{\sqrt{x\left(x-4 M_{H}^{2} / s\right)}}{512 \pi^{4}} \\
& \times\left(\frac{1}{1+y}\right)_{+} f_{g q}\left(x, y, \theta_{1}, \theta_{2}\right) .
\end{aligned}
$$

Again, we already included the counterterms in the definition of $\hat{\sigma}_{q g}^{(c+)}$ and $\hat{\sigma}_{g q}^{(c-)}$. Finally, for the quark-antiquark subprocess we have 


$$
\hat{\sigma}_{q \bar{q}}^{b}=\int d \cos \theta_{1} d \theta_{2} d y \frac{\sqrt{x\left(x-4 M_{H}^{2} / s\right)}}{512 \pi^{4}} f_{q \bar{q}}\left(x, y, \theta_{1}, \theta_{2}\right) .
$$

The expressions for $f_{q g}, f_{g q}$, and $f_{q \bar{q}}$ can be found in the Supplemental Material [28].

Summarizing, Eqs. (3), (14), (16), and (17) contain all the contributions to the partonic cross section up to NNLO accuracy. We find agreement with Ref. [16] with respect to the NLO results. (We notice that the exact LO is taken into account in a slightly different way in Ref. [16]. The numerical effect is, anyway, small.)

Phenomenology.-We present, here, the phenomenological results for the LHC. In all cases, we use the MSTW2008 [31] sets of parton distributions and QCD coupling at each corresponding order. The bands are obtained by varying independently the factorization and renormalization scales in the range $0.5 Q \leq \mu_{F}, \mu_{R} \leq 2 Q$, with the constraint $0.5 \leq \mu_{F} / \mu_{R} \leq 2$. We recall that we always normalize our results with the exact top- and bottom-mass dependence at LO. We use $M_{H}=$ $126 \mathrm{GeV}, M_{t}=173.18 \mathrm{GeV}$, and $M_{b}=4.75 \mathrm{GeV}$.

Given that at one-loop order the corrections to the effective vertex $g g H H$ are the same as those of $g g H$, we will assume for the phenomenological results that $C_{H H}^{(2)}=$ $C_{H}^{(2)}$. We analyzed the impact of this still unknown coefficient varying its value in the range $0 \leq C_{H H}^{(2)} \leq 2 C_{H}^{(2)}$ and found a variation in the total cross section of less than $2.5 \%$.

In Fig. 2, we show the hadronic cross section for the LHC as a function of the Higgs pair invariant mass, for a c.m. energy $E_{\text {c.m. }}=\sqrt{s_{H}}=14 \mathrm{TeV}$, at LO, NLO, and NNLO accuracy. We can observe that it is only at this order that the first sign of convergence of the perturbative series appears, finding a nonzero overlap between the NLO and NNLO bands. Second order corrections are sizeable,

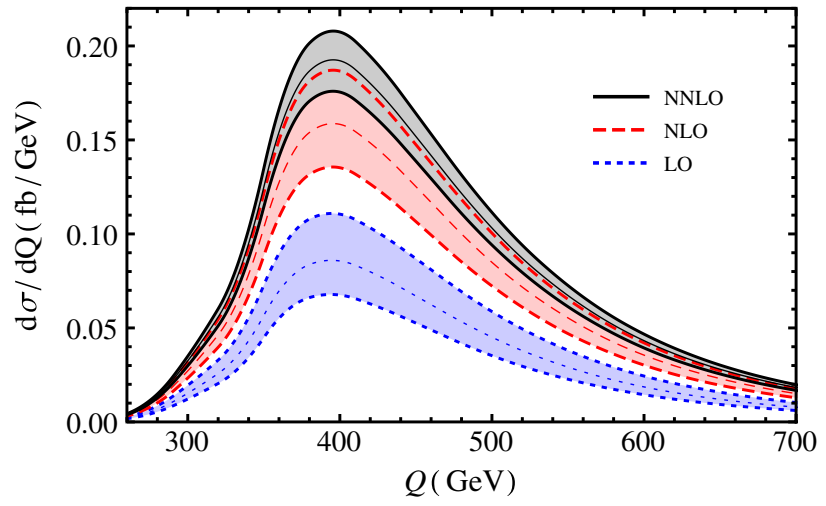

FIG. 2 (color online). Higgs pair invariant mass distribution at LO (dotted blue), NLO (dashed red), and NNLO (solid black) for the LHC at c.m. energy $E_{\text {c.m. }}=14 \mathrm{TeV}$. The bands are obtained by varying $\mu_{F}$ and $\mu_{R}$ in the range $0.5 Q \leq \mu_{F}, \mu_{R} \leq 2 Q$ with the constraint $0.5 \leq \mu_{F} / \mu_{R} \leq 2$. this is noticeable already at the level of the total inclusive cross sections

$$
\begin{gathered}
\sigma_{\mathrm{LO}}=17.8_{-3.8}^{+5.3} \mathrm{fb}, \quad \sigma_{\mathrm{NLO}}=33.2_{-4.9}^{+5.9} \mathrm{fb}, \\
\sigma_{\mathrm{NNLO}}=40.2_{-3.5}^{+3.2} \mathrm{fb},
\end{gathered}
$$

where the uncertainty arises from the scale variation. The increase with respect to the NLO result is then of $\mathcal{O}(20 \%)$, and the $K$ factor with respect to the LO prediction is about $K_{\mathrm{NNLO}}=2.3$. The scale dependence is clearly reduced at this order, resulting in a variation of about $\pm 8 \%$ around the central value, compared to a total variation of $\mathcal{O}( \pm 20 \%)$ at NLO.

In Fig. 3, we present the total cross section as a function of the c.m. energy $E_{\text {c.m. }}$, in the range from 8 to $100 \mathrm{TeV}$. We can observe that the size of the perturbative corrections is smaller as the c.m. energy increases. Again, in the whole range of energies the scale dependence is substantially reduced when we consider the second order corrections.

In Table I, we show the value of the NNLO cross section for $E_{\text {c.m. }}=8,14,33$, and $100 \mathrm{TeV}$. We considered three different sources of theoretical uncertainties: missing higher orders in the QCD perturbative expansion, which are estimated by the scale variation as indicated before, uncertainties in the determination of the parton distributions, and strong coupling. To estimate the parton flux and coupling constant uncertainties, we used the MSTW2008 90\% C.L. error PDF sets [32], which are known to provide very close results to the PDF4LHC working group recommendation for the envelope prescription [33]. We observe that nonperturbative and perturbative uncertainties are of the same order.

The ratio between NNLO and NLO predictions as a function of the c.m. energy is quite flat. In order to ease the use of our NNLO results, we provide the following approximated analytic expression for the $K$ factor, valid in the range $8 \mathrm{TeV} \leq E_{\text {c.m. }} \leq 100 \mathrm{TeV}$

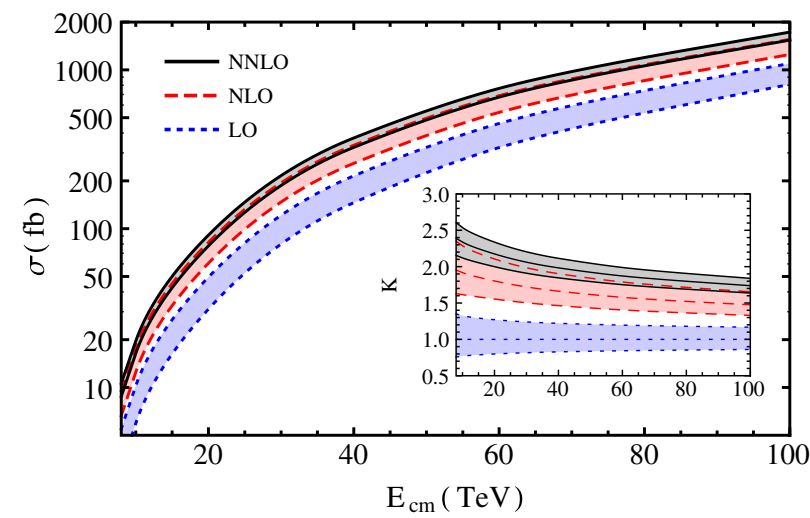

FIG. 3 (color online). Total cross section as a function of the c.m. energy $E_{\text {c.m. }}$ for the LO (dotted blue), NLO (dashed red), and NNLO (solid black) prediction. The bands are obtained by varying $\mu_{F}$ and $\mu_{R}$ as indicated in the main text. The inset plot shows the corresponding $K$ factors. 
TABLE I. Total cross section as a function of the c.m. energy at NNLO accuracy. We use the exact LO prediction to normalize our results. The different sources of theoretical uncertainties are discussed in the main text.

\begin{tabular}{lcccc}
\hline \hline$E_{\text {c.m. }}$ & $8 \mathrm{TeV}$ & $14 \mathrm{TeV}$ & $33 \mathrm{TeV}$ & $100 \mathrm{TeV}$ \\
\hline$\sigma_{\text {NNLO }}$ & $9.76 \mathrm{fb}$ & $40.2 \mathrm{fb}$ & $243 \mathrm{fb}$ & $1638 \mathrm{fb}$ \\
Scale [\%] & $+9.0-9.8$ & $+8.0-8.7$ & $+7.0-7.4$ & $+5.9-5.8$ \\
PDF [\%] & $+6.0-6.1$ & $+4.0-4.0$ & $+2.5-2.6$ & $+2.3-2.6$ \\
PDF $+\alpha_{\mathrm{S}}[\%]$ & $+9.3-8.8$ & $+7.2-7.1$ & $+6.0-6.0$ & $+5.8-6.0$ \\
\hline \hline
\end{tabular}

$$
\begin{aligned}
\frac{\sigma_{\mathrm{NNLO}}}{\sigma_{\mathrm{NLO}}}= & 1.149-0.326\left(\frac{E_{\text {c.m. }}}{1 \mathrm{TeV}}\right)^{-1} \\
& +0.327\left(\frac{E_{\text {c.m. }}}{1 \mathrm{TeV}}\right)^{-1 / 2},
\end{aligned}
$$

which runs from 1.22 at $8 \mathrm{TeV}$ to 1.18 at $100 \mathrm{TeV}$. On the other hand, the ratio between NNLO and LO runs from 2.39 to 1.74 in the same range of energies, and can be parametrized by the following expression:

$$
\frac{\sigma_{\mathrm{NNLO}}}{\sigma_{\mathrm{LO}}}=1.242-7.17\left(\frac{E_{\text {c.m. }}}{1 \mathrm{TeV}}\right)^{-1}+5.77\left(\frac{E_{\text {c.m. }}}{1 \mathrm{TeV}}\right)^{-1 / 2} .
$$

Finally, the total scale variation at NNLO is approximately given by $\pm p\left(E_{\text {c.m. }}\right) \%$, with

$$
p\left(E_{\text {c.m. }}\right)=4.07-9.8\left(\frac{E_{\text {c.m. }}}{1 \mathrm{TeV}}\right)^{-1}+18.6\left(\frac{E_{\text {c.m. }}}{1 \mathrm{TeV}}\right)^{-1 / 2} .
$$

In this case, we have $\pm 9.4 \%$ and $\pm 5.8 \%$ at 8 and $100 \mathrm{TeV}$, respectively.

It is worth noticing that the soft-virtual approximation presented in [17] gives an extremely accurate prediction for the NNLO cross section, overestimating for example the $E_{\text {c.m. }}=14 \mathrm{TeV}$ result by less than $2 \%$. As expected, this approximation works even better than for single Higgs production, due to the larger invariant mass of the final state.

This work was supported in part by UBACYT, CONICET, ANPCyT, and the Research Executive Agency (REA) of the European Union under the Grant Agreement No. PITN-GA-2010-264564 (LHCPhenoNet).

*deflo@df.uba.ar jmazzi@df.uba.ar

[1] G. Aad et al. (ATLAS Collaboration), Phys. Lett. B 716, 1 (2012).

[2] S. Chatrchyan et al. (CMS Collaboration), Phys. Lett. B 716, 30 (2012).

[3] F. Englert and R. Brout, Phys. Rev. Lett. 13, 321 (1964); P. W. Higgs, Phys. Lett. 12, 132 (1964); Phys. Rev. Lett. 13, 508 (1964).

[4] U. Baur, T. Plehn, and D. L. Rainwater, Phys. Rev. D 67, 033003 (2003).
[5] M. J. Dolan, C. Englert, and M. Spannowsky, J. High Energy Phys. 10 (2012) 112.

[6] A. Papaefstathiou, L. L. Yang, and J. Zurita, Phys. Rev. D 87, 011301 (2013).

[7] J. Baglio, A. Djouadi, R. Grober, M. M. Muhlleitner, J. Quevillon, and M. Spira, J. High Energy Phys. 04 (2013) 151.

[8] U. Baur, T. Plehn, and D. L. Rainwater, Phys. Rev. D 69, 053004 (2004).

[9] M. J. Dolan, C. Englert, and M. Spannowsky, Phys. Rev. D 87, 055002 (2013).

[10] F. Goertz, A. Papaefstathiou, L. L. Yang, and J. Zurita, J. High Energy Phys. 06 (2013) 016.

[11] D. Y. Shao, C. S. Li, H. T. Li, and J. Wang, J. High Energy Phys. 07 (2013) 169.

[12] M. Gouzevitch, A. Oliveira, J. Rojo, R. Rosenfeld, G. Salam, and V. Sanz, J. High Energy Phys. 07 (2013) 148.

[13] E. W. N. Glover and J. J. van der Bij, Nucl. Phys. B309, 282 (1988).

[14] O. J.P. Eboli, G. C. Marques, S.F. Novaes, and A. A. Natale, Phys. Lett. B 197, 269 (1987).

[15] T. Plehn, M. Spira, and P. M. Zerwas, Nucl. Phys. B479, 46 (1996); B531, 655(E) (1998).

[16] S. Dawson, S. Dittmaier, and M. Spira, Phys. Rev. D 58, 115012 (1998).

[17] D. de Florian and J. Mazzitelli, Phys. Lett. B 724, 306 (2013).

[18] D. de Florian and J. Mazzitelli, J. High Energy Phys. 12 (2012) 088.

[19] J. Grigo, J. Hoff, K. Melnikov, and M. Steinhauser, Nucl. Phys. B875, 1 (2013).

[20] M. Kramer, E. Laenen, and M. Spira, Nucl. Phys. B511, 523 (1998)

[21] K. G. Chetyrkin, B. A. Kniehl, and M. Steinhauser, Phys. Rev. Lett. 79, 353 (1997).

[22] A. Djouadi, M. Spira, and P. M. Zerwas, Phys. Lett. B 264, 440 (1991).

[23] R. V. Harlander and W. B. Kilgore, Phys. Rev. Lett. 88, 201801 (2002).

[24] C. Anastasiou and K. Melnikov, Nucl. Phys. B646, 220 (2002).

[25] V. Ravindran, J. Smith, and W. L. van Neerven, Nucl. Phys. B665, 325 (2003).

[26] T. Hahn, Comput. Phys. Commun. 140, 418 (2001).

[27] R. Mertig, M. Bohm, and A. Denner, Comput. Phys. Commun. 64, 345 (1991).

[28] See Supplemental Material at http://link.aps.org/ supplemental/10.1103/PhysRevLett.111.201801 for more details about the phase space parametrization and the explicit expressions of $f_{g g}, f_{q g}, f_{g q}$, and $f_{q \bar{q}}$.

[29] S. Frixione, Z. Kunszt, and A. Signer, Nucl. Phys. B467, 399 (1996).

[30] S. Frixione, Nucl. Phys. B410, 280 (1993).

[31] A. D. Martin, W. J. Stirling, R. S. Thorne, and G. Watt, Eur. Phys. J. C 63, 189 (2009).

[32] A. D. Martin, W. J. Stirling, R. S. Thorne, and G. Watt, Eur. Phys. J. C 64, 653 (2009).

[33] M. Botje, J. Butterworth, A. Cooper-Sarkar, A. de Roeck, J. Feltesse, S. Forte, A. Glazov, and J. Huston et al., arXiv:1101.0538. 